\title{
Near infrared throughput and stray light measurements of diffraction gratings for ELT-HARMONI
}

\author{
John I. Capone ${ }^{\mathrm{a}}$, Myriam Rodrigues ${ }^{\mathrm{a}}$, Fraser Clarke ${ }^{\mathrm{a}}$, Andrew Earle ${ }^{\mathrm{b}}$, Thomas Foster ${ }^{\mathrm{a}}$, Andrea \\ Hidalgo Valadez ${ }^{\mathrm{a}}$, Ian Lewis ${ }^{\mathrm{a}}$, James Lynn ${ }^{\mathrm{a}}$, Kieran O'Brien ${ }^{\mathrm{c}}$, Matthias Tecza ${ }^{\mathrm{a}}$, Niranjan \\ Thatte $^{\mathrm{a}}$, and Ian Tosh ${ }^{\mathrm{b}}$ \\ ${ }^{a}$ University of Oxford, The Denys Wilkinson Building, Keble Road, Oxford, OX1 3RH, UK \\ ${ }^{\mathrm{b}}$ Rutherford Appleton Laboratory, Harwell Campus, Didcot, OX11 0QX, UK \\ ${ }^{c}$ Durham University, South Road, Durham, DH1 3LE, UK
}

\begin{abstract}
The High Angular Resolution Monolithic Optical and Near-infrared Integral field spectrograph (HARMONI) will be one of the instruments installed on ESO's 39-meter Extremely Large Telescope (ELT) at first light. The instrument will operate from $0.47-2.45 \mu \mathrm{m}$ with $\Delta \lambda / \lambda=3,000-17,000$. On-sky spatial pixels (spaxels) are divided between four spectrographs, each equipped with 11 transmission diffraction gratings to cover the ranges of wavelengths and spectral resolutions. These spectrographs will be cooled to $\sim 140 \mathrm{~K}$ to decrease thermal radiation at longer wavelengths.

In all configurations, the diffraction grating will lose a greater fraction of scientific light than any other single optic in the instrument. Additionally, manufacturers are often unable to measure the fraction of transmitted light at HARMONI's longest wavelengths. For these reasons, we have developed a setup to measure the efficiencies of transmission diffraction gratings across HARMONI's bandpass. The setup uses modulated signals, a single detector, and a lock-in amplifier to minimize sources of systematic errors. A modified version of this setup may be used to measure stray light. These setups and initial results are presented.
\end{abstract}

Keywords: metrology, near-infrared, spectrophotometer, diffraction grating, efficiency, stray light, lock-in amplifier, HARMONI, ELT

\section{INTRODUCTION}

The HARMONI instrument will include the transmission diffraction gratings described in Table 1. The observed field on the sky is divided between four identical spectrograph subsystems, and so four copies of each grating are required for a total of 44 gratings. The gratings will be located at the pupil and and must have a clear aperture of $\gtrsim 150 \mathrm{~mm}$, depending on the incident angle. Each grating is required to transmit $>70 \%$ of the incident light into the first diffractive order over the central $80 \%$ of wavelengths within its bandpass. Additionally, stray light (e.g. from scattering, periodic grating errors, etc.) must be understood and minimized.

\section{SETUPS}

\subsection{Throughput Measurement}

Commercially available spectrophotometers can precisely measure the fraction of light transmitted through a medium (e.g. Figure 1). These devices minimize systematic errors by splitting a light source into two beams ("signal" and "normalizing" paths), modulating these beams at different frequencies, and passing both beams to a common detector where the signals are recovered by lock-in amplifiers, such as the setup shown in Figure 2. The signal beam is passed through a sample (e.g. a filter) to measure the fraction of light transmitted relative to the normalizing path, and an absolute measurement is obtained by taking calibration measurements without

Further author information: (Send correspondence to J.I.C.)

J.I.C.: E-mail: john.capone@physics.ox.ac.uk, Telephone: +44 (0)1865 283911 
Table 1. Each of the four spectrographs in HARMONI can be configured with one of the following transmission gratings.

\begin{tabular}{l|l|l|l|l|l} 
Name & Band & $\lambda_{\min }(\mu \mathrm{m})$ & $\lambda_{\max }(\mu \mathrm{m})$ & Lines $/ \mathrm{mm}$ & Incident angle (deg.) \\
\hline \hline VIS & VRI & 0.463 & 0.820 & 451 & 8.413 \\
\hline LR1 & izJ & 0.810 & 1.369 & 287 & 9.109 \\
\hline LR2 & HK & 1.450 & 2.450 & 160 & 9.109 \\
\hline MR1 & iz & 0.830 & 1.050 & 676 & 18.726 \\
\hline MR2 & $\mathrm{J}$ & 1.046 & 1.324 & 536 & 18.726 \\
\hline MR3 & $\mathrm{H}$ & 1.435 & 1.815 & 391 & 18.726 \\
\hline MR4 & $\mathrm{K}$ & 1.951 & 2.469 & 287 & 18.726 \\
\hline HR1 & $\mathrm{z}$ & 0.827 & 0.903 & 1444 & 39.120 \\
\hline HR2 & $\mathrm{J}_{\text {high }}$ & 1.190 & 1.300 & 1003 & 39.120 \\
\hline HR3 & $\mathrm{H}_{\text {high }}$ & 1.534 & 1.676 & 778 & 39.120 \\
\hline HR4 & $\mathrm{K}_{\text {high }}$ & 2.093 & 2.287 & 570 & 39.120
\end{tabular}

a sample in place (i.e. where $100 \%$ of incident light is transmitted). Since both beams originate from the same light source, any variability in the source is common to both paths. Additionally, by converting both beams into electrical signals using the same detector, any variations in the response of the detector are common to both signals. This type of setup also greatly reduces noise in the measurements by filtering out the two signals at known frequencies and phases.

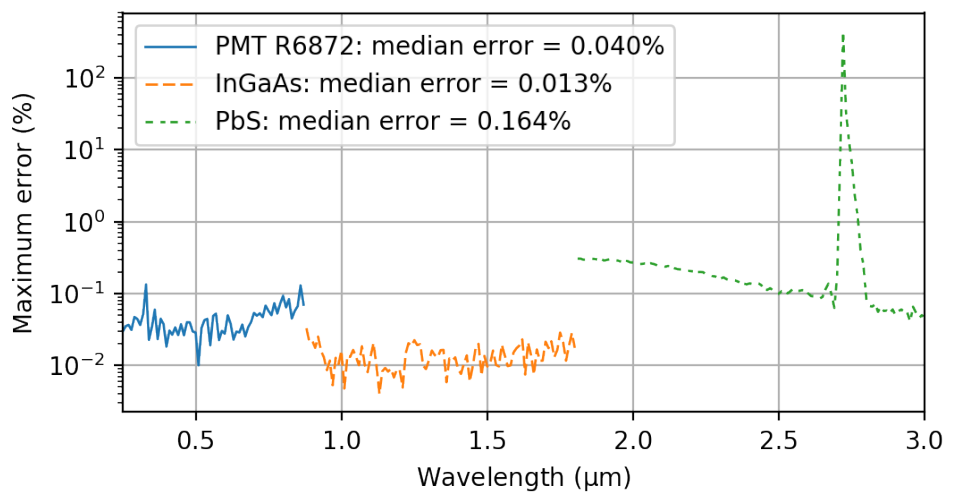

Figure 1. The errors plotted above are calculated from measurements taken with a Perkin Elmer LAMBDA 1050. The maximum error is the maximum difference of two measurements at a given wavelength under the same conditions from a set of six measurement runs.

Unfortunately off-the-shelf spectrophotometers are not suitable for measuring the diffraction efficiencies $(\eta \triangleq$ $\left.I_{\text {in }} / I_{\text {out }}\right)$ of HARMONI's gratings. First, the measurement chambers are too small and the paths are too close to permit measurements across the clear apertures of the gratings. Second, the gratings will deflect the signal path, and the angle of the deflection will depend on the wavelength of the incident light $(\lambda)$ and on the diffraction order $(m)$ being measured,

$$
\left(n_{2} \sin \theta_{\text {out }}-n_{1} \sin \theta_{\text {in }}\right) \cos \phi=\frac{m \lambda}{\Lambda},
$$

where $n_{1}$ and $n_{2}$ are the refractive indices before and after the grating $\left(n_{1}=n_{2} \simeq 1\right)$ and $\Lambda$ is the groove 


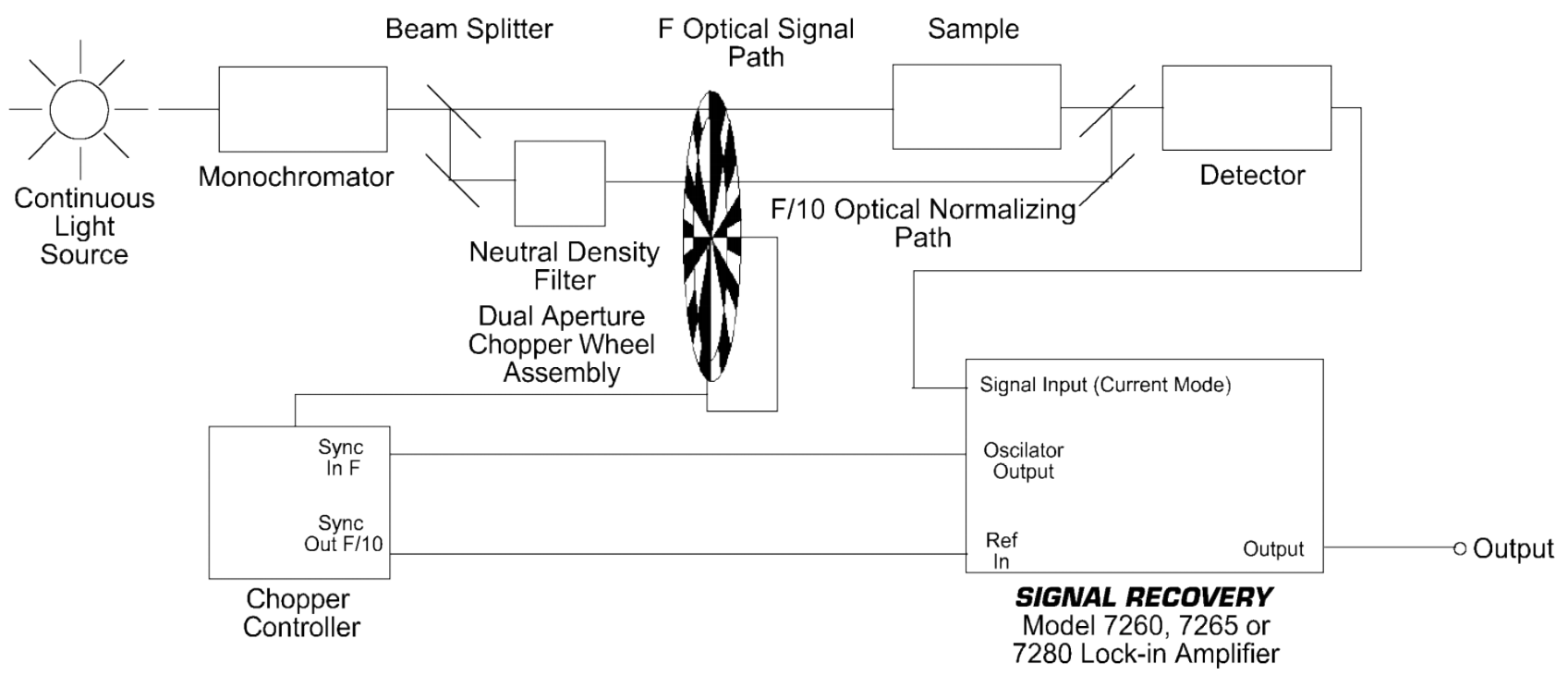

Figure 2. This spectrophotometer setup from Figure 6 of Reference 1 uses a single detector. By producing two beams with the same source and detecting both with the same detector, variations in the lamp intensity and detector response are common to both paths. Measurements with a sample in position yield the relative throughput of the sample. Absolute measurements are obtained by removing the sample to determine the relative intensities of the two beams.

period of the grating in the same units as $\lambda$. The angles of the incident and outgoing beams $\left(\theta_{\text {in }}, \theta_{\text {out }}\right.$, and $\left.\phi\right)$ are the defined in Figure 3.

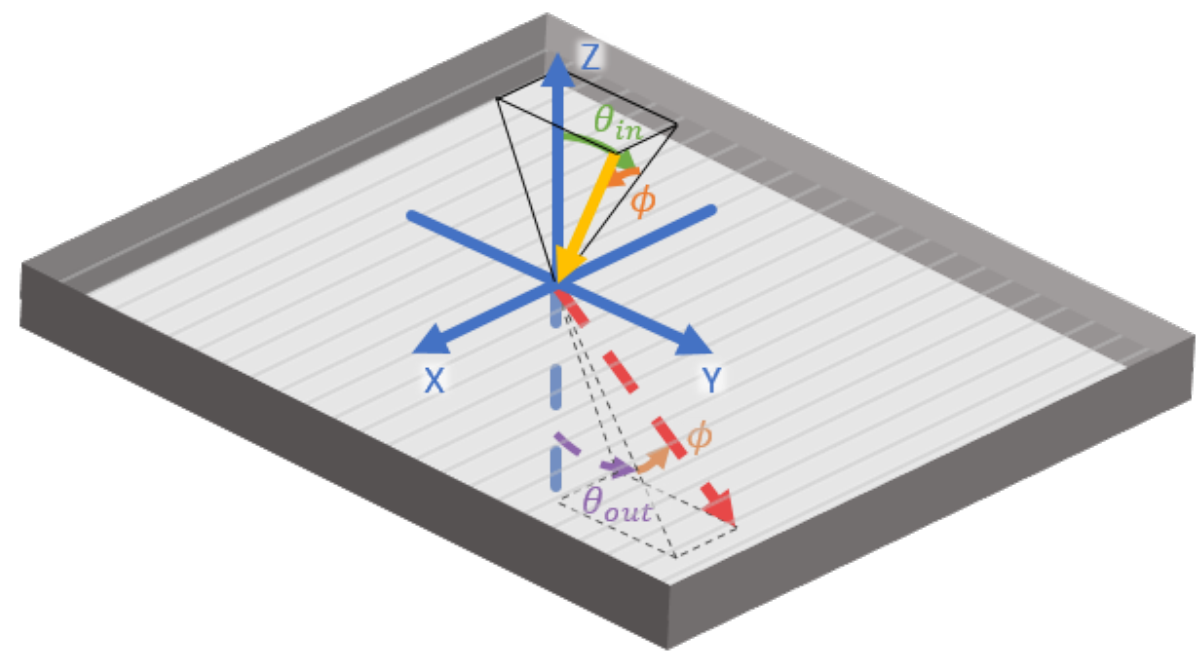

Figure 3. The angles $\theta_{\text {in }}, \theta_{\text {out }}$, and $\phi$ from Equation 1 are defined above relative to the local coordinate system of a diffraction grating. The yellow arrow shows an incident ray while the red arrow shows the diffracted ray. Lines below the grating are dashed.

Rather than attempting to modify one of these instruments, we have decided to develop a more flexible setup. Figure 4 presents an overview of our planned test bench. We use a quartz tungsten halogen (QTH) lamp and a monochromator to create a monochromatic source for the setup. The grating drive and filter wheel of the monochromator are motorized so that the wavelength can be selected by control software.

The output slit of the monochromator is collimated by an achromatic doublet (L1), as shown in Figure 5 . A pupil stop consisting of two circular apertures is placed immediately after this lens. These two apertures are 


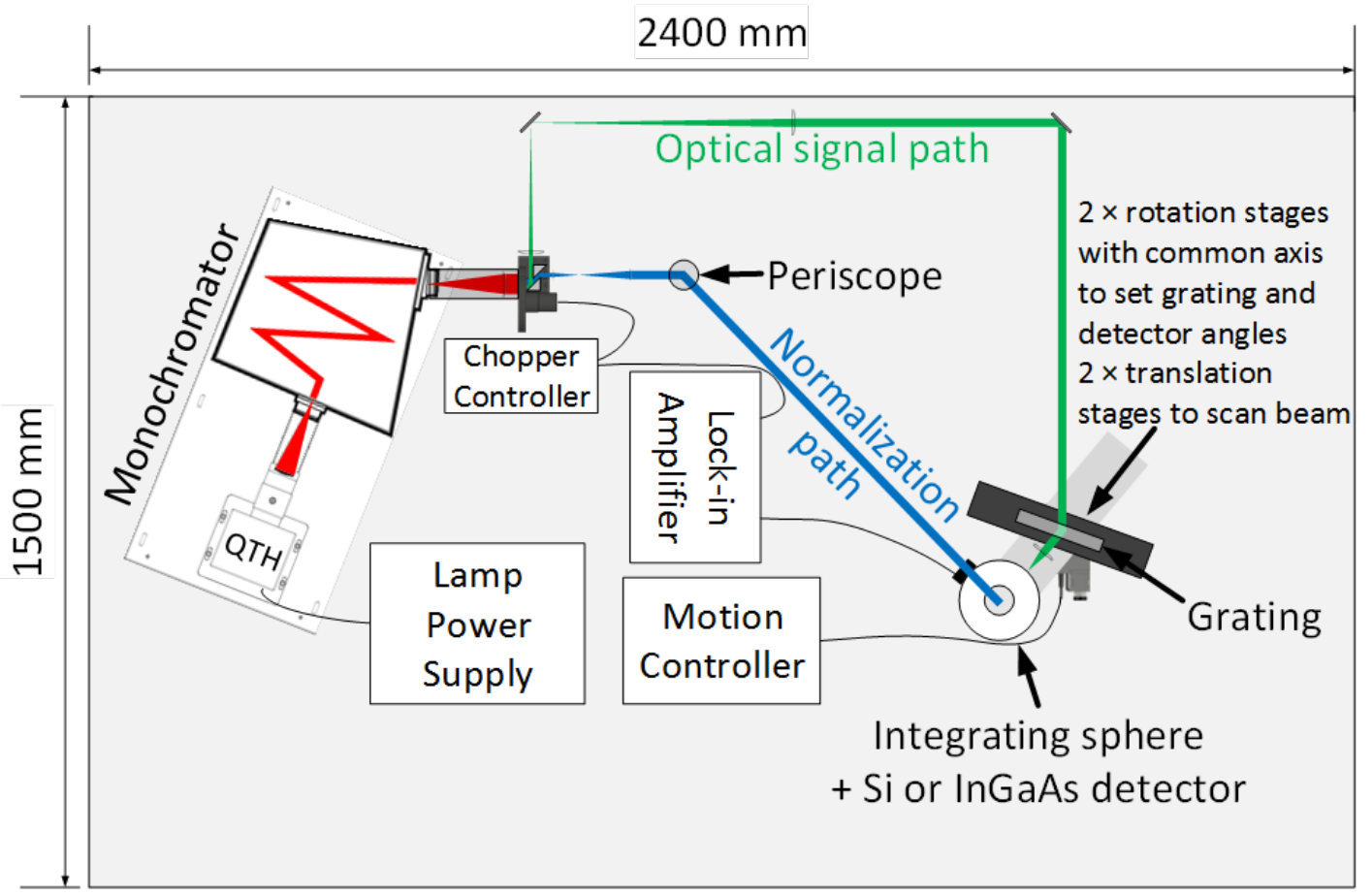

Figure 4. The planned setup for measuring diffraction grating throughput is based on the setup shown in Figure 2 and described in Reference 1. The monochromator allows the control software to select wavelengths between $0.4 \mu \mathrm{m}$ and 2.5 $\mu \mathrm{m}$. The output beam is divided into two beams which are modulated at the $5^{\text {th }}$ and $7^{\text {th }}$ harmonics to form the signal and normalization paths. Both beams are directed into an integrating sphere, where the light is combined and detected. The modulated signals are then recovered using a lock-in amplifier capable of filtering at two harmonics of a reference frequency.

aligned with the inner and outer rings of a dual aperture chopper wheel, similar to the one shown in Figure 2. The inner ring of the chopper wheel has 5 openings, while the outer ring has 7 openings, both at a $50 \%$ duty cycle. By rotating this wheel at a set frequency $(f)$, the two beams are modulated at the $5^{\text {th }}$ and $7^{\text {th }}$ harmonics of this frequency.

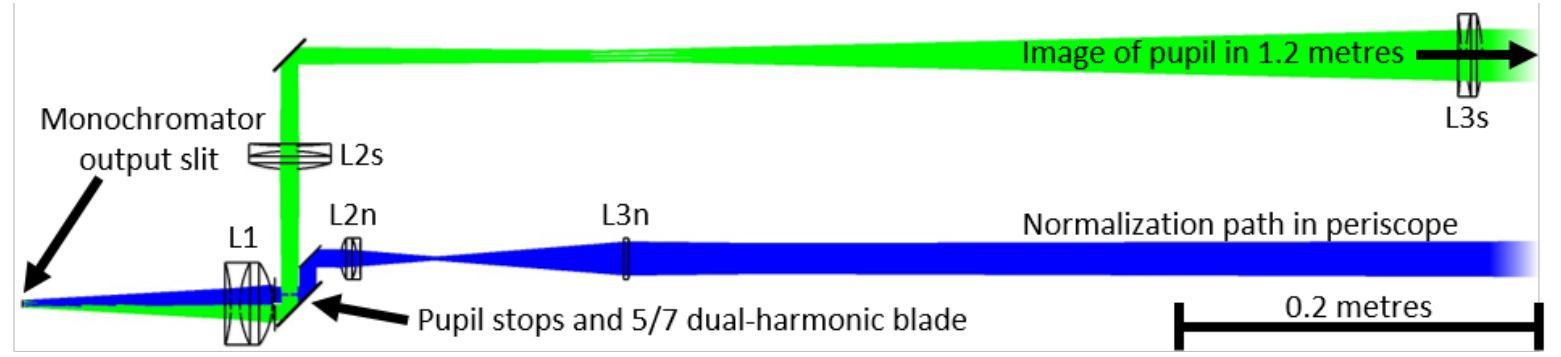

Figure 5. This optical layout starts with the output slit of the monochromator and shows how beams are formed for the signal and normalization paths. The signal path is formed by the lower pupil stop passing through the $5^{\text {th }}$ harmonic aperture of the chopper blade, while the normalization path is formed by the upper aperture and is modulated at the $7^{\text {th }}$ harmonic of the reference frequency.

The two beams are then separated using fold mirrors. Two lenses (L2n and L3n in Figure 5) are used to minimize the diameter of the normalization path beam to avoid vignetting as it travels through the periscope. The periscope raises the beam above the rest of the setup and directs the beam into the integrating sphere as 
the sphere is rotated about the grating being measured.

The signal path is focused and re-collimated using two achromatic doublets (L2s and L3s in Figure 5) to set the beam size and the range of angles incident on the grating. Figure 6 shows the relative illumination of the 16 $\mathrm{mm}$ diameter signal path beam, assuming the pupil stop is uniformly illuminated by the monochromator.

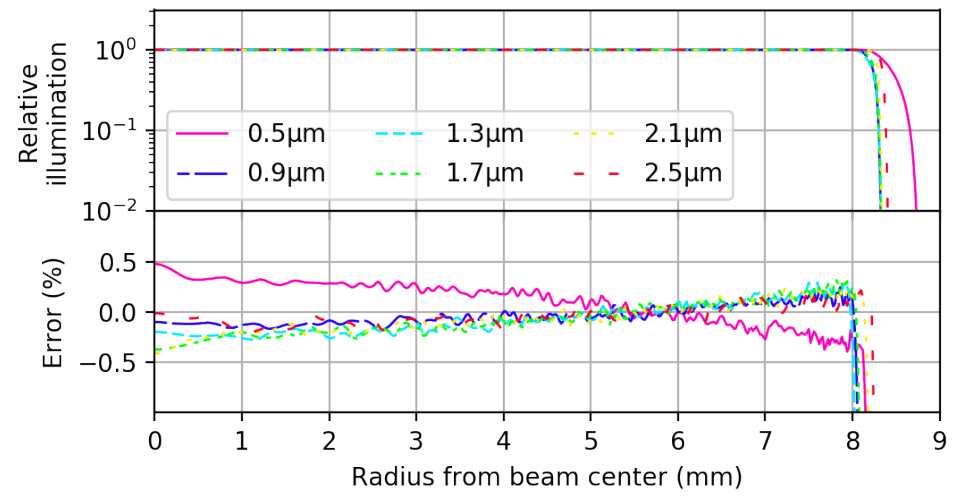

Figure 6. Inhomogeneous illumination increases the uncertainty of which sub-aperture of the grating is being measured since the weighting is not uniform. The top plot shows the relative illumination of the signal path's beam at the grating calculated for the design shown in Figure 5. The bottom plot shows the percent deviation from the median value. These plots assume that the pupil stop is uniformly illuminated.

In addition to the selection of focal lengths of lenses L2s and L3s, the range of angles incident on the grating depends on the width of the monochromator slits. Figure 7 shows how this range varies from the minimum slit width $(\sim 50 \mu \mathrm{m})$ to the maximum slit width $(3 \mathrm{~mm})$. Because the efficiency of a grating depends on the incident angle, a wider range of angles increases the error of our measurement for a given nominal angle. Figure 8 shows rigorous coupled-wave analysis (RCWA) calculations for a model of the LR2 volume phase holographic grating (VPHG). This shows that although the efficiency can differ significantly at the extreme angles in these ranges, the efficiency averaged over this range is much closer to that at the nominal incidence angle.

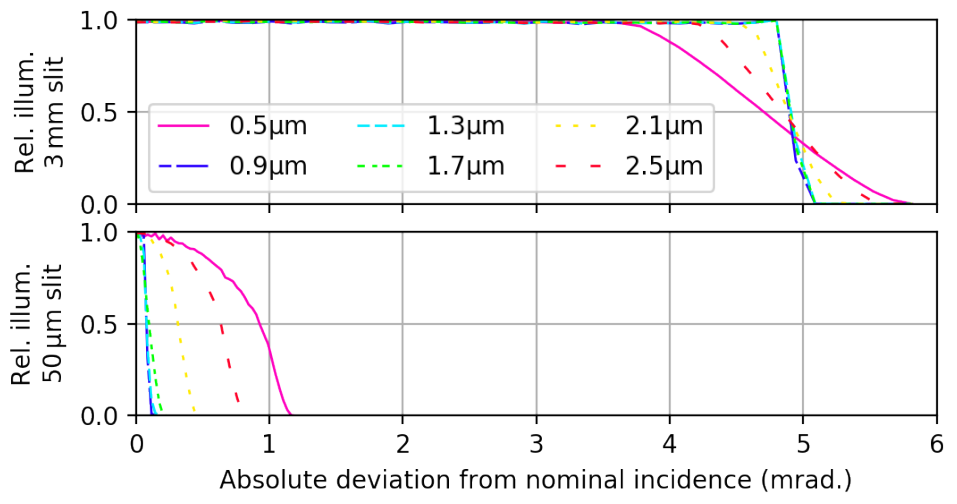

Figure 7. The distribution of intensity versus incident angles in the collimated beam depends on the selection of L2s and L3s from Figure 5 and the width of the monochromator slit. The top plot shows the distributions across the bandpass when the slit is fully open, while the bottom plot shows these distributions when the slit is very narrow. Figure 8 shows how these deviations from the nominal incidence impact the measured throughput.

As shown in Figure 4, the grating undergoing measurement is mounted on two motorized translation stages which will move the grating in axes orthogonal to its normal. By translating the grating, different positions 


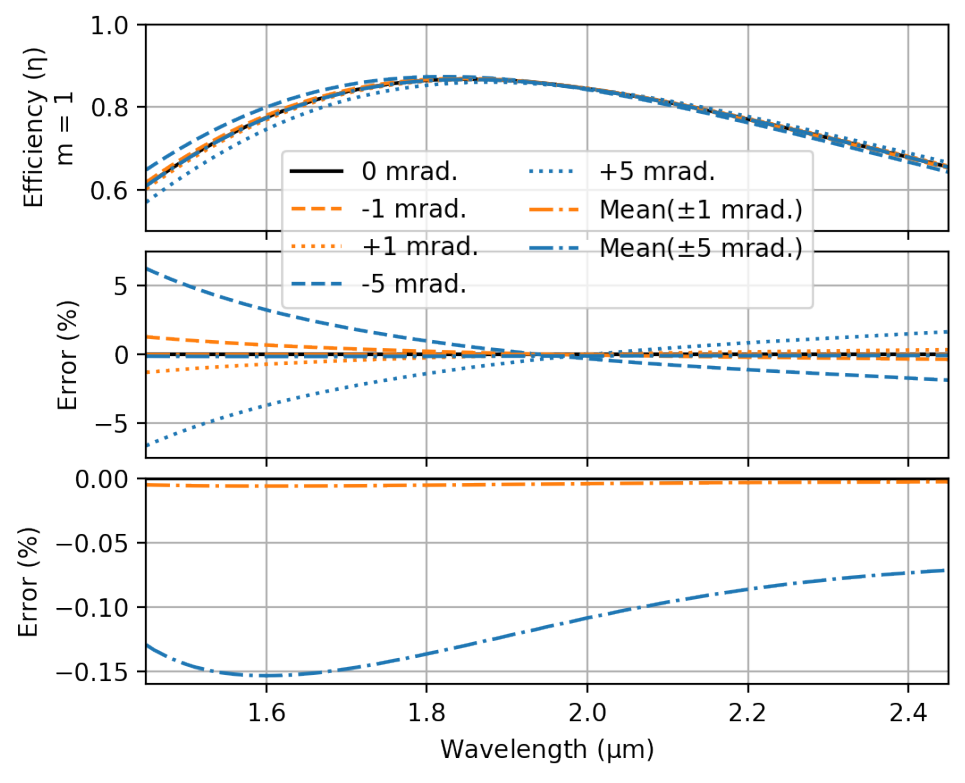

Figure 8. The top plot shows calculated efficiencies for the LR2 grating at incident angles typical of the distributions from Figure 7. The middle plot shows the percent deviation of the throughput at a given angle relative to that of the nominal incidence. The bottom plot shows the errors of measurements which average over a range of angles. These errors are low since the distributions of incident angles are symmetrical about the nominal angle.

within its clear aperture can be measured. These stages and the grating are all mounted on a motorized rotation stage to set the incident angle of the beam. A second rotation stage moving about the same axis moves an arm holding a lens and an integrating sphere. The lens focuses the beam into the sphere where the light from both paths is combined. The optical signals are detected by a silicon ( $\mathrm{Si}$, for $\lambda \lesssim 1.1 \mu \mathrm{m}$ ) or indium gallium arsenide (InGaAs, for $\lambda \gtrsim 1.1 \mu \mathrm{m}$ ) detector.

In addition to measurements with a grating in place, measurements are required without a grating to determine absolute efficiencies. These calibration runs must include measurements with Nd filters at the grating position to determine the response of the detector over a range of intensities.

\subsection{Stray Light Measurement}

Ideally, all monochromatic light with a particular incidence onto a grating will leave the grating at the same angle, however real gratings will produce some amount of stray light. We can use a modified version of the setup described in Section 2.1 to measure this stray light.

First, the monochromator will be replaced with a laser to increase the available monochromatic signal. Second, a slit will be positioned on the optical axis at the focus of the lens in the signal path preceding the integrating sphere. The slit will be aligned with its long axis parallel with the axes of rotation of the motorized stages under the grating and the width of the slit will be matched to the focused spot size of the lens.

The amount of stray light versus angle is then measured by rotating the lens, slit, and integrating sphere about the grating. This measurement is compared with calibration measurements to determine the excess stray light produced by the grating. 


\section{INITIAL RESULTS}

The current setup, shown in Figure 9, has been used to understand and develop the setups described in Section 2 .

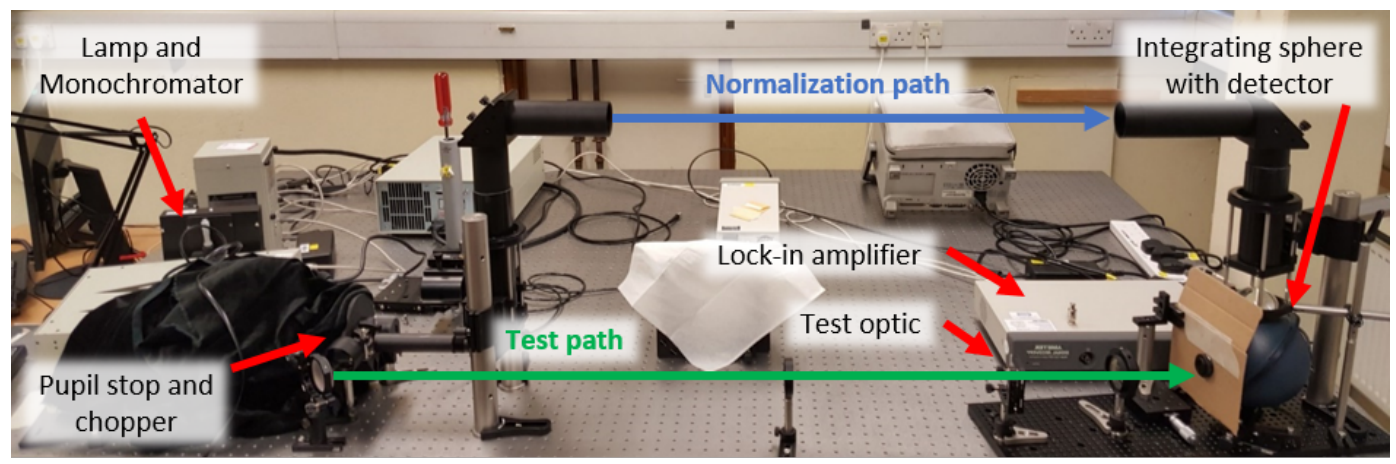

Figure 9. The current bench follows the layout presented in Figure 4 without the rotation and translation stages used to automate the motion of the grating and integrating sphere. Additionally, the periscope must be improved to maintain the alignment between the two ends.

As mentioned previously, we reduce the noise in our measurements by recovering the modulated signals with a lock-in amplifier. This improvement is demonstrated in Figure 10 where reference frequencies are plotted versus the measured ratios of the two paths and the maximum deviation from this value. The maximum deviation is fairly constant at frequencies greater than $\sim 20 \mathrm{~Hz}$ where the modulation frequencies are $>100 \mathrm{~Hz}$ and $>140 \mathrm{~Hz}$. This is likely due to $1 / \mathrm{f}$ noise no longer being dominant.

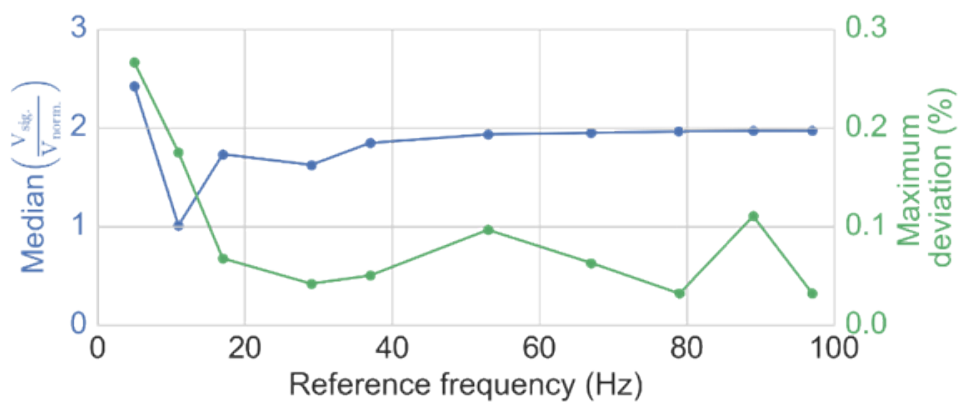

Figure 10. Measurements were taken at $500 \mathrm{~nm}$ while varying the reference frequency. The median of 1000 measurements is plotted on the left axis, while the maximum deviation from this median is plotted on the right.

We have measured the zeroth order efficiency of a transmission grating to test the performance of our setup. Figure 11 compares measurements taken with our setup to measurements from a Perkin Elmer LAMBDA 1050 and to typical values provided by the manufacturer.

Several parts of our test bench must be completed to enable automated measurements of all 44 HARMONI gratings. The periscope must mechanically maintain the alignment between the two ends of the normalization path so that we can measure non-zero diffraction orders. Additionally, we must add an extended range InGaAs detector so that we can measure from $1.1 \mu \mathrm{m}$ to $2.45 \mu \mathrm{m}$.

\section{REFERENCES}

[1] "Low level optical detection using lock-in amplifier techniques," tech. rep., Oak Ridge, Tennessee (2008). 


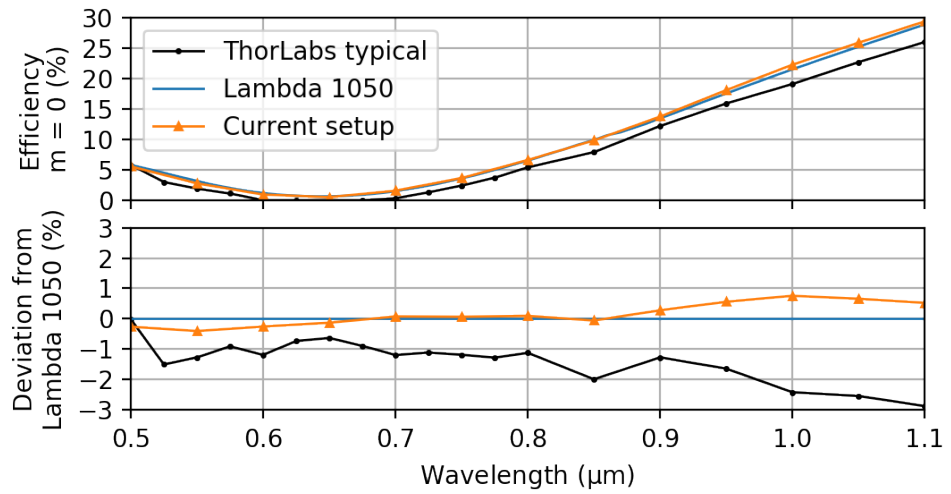

Figure 11. Measurements were taken of the zeroth order of a ThorLabs GT150-03 transmission grating as part of the development of the test bench. The black curve was provided by the manufacturer and shows typical values, not those of this specific grating. The blue curve shows measurements taken with the same Perkin Elmer LAMBDA 1050 spectrophotometer as used in Figure 1. The deviation between measurements taken with our setup and those taken with the LAMBDA 1050 may be due to the wavelength calibration of our monochromator. 